\title{
Estrategias de desarrollo territorial y turismo contra la despoblación de las áreas rurales en Cerdeña (Italia)
}

\author{
Salvatore Lampreu*
}

Università degli Studi di Sassari - Dipartimento di Scienze Umanistiche e Sociali (DUMAS) - Italia

Recibido: 30 de mayo de 2020 / Aceptado: 8 de julio de 2020

\begin{abstract}
Resumen
La despoblación de las áreas rurales es un problema para numerosas regiones europeas, incluida Cerdeña (Italia), donde hay varias poblaciones en peligro de desaparecer. Se han llevado a cabo diferentes políticas para reforzar su competitividad, y el presente trabajo pretende subrayar las oportunidades ofrecidas por distintas estrategias. Se analizarán, por tanto, los principales proyectos de desarollo territorial promovidos en Cerdeña y el papel del turismo en la búsqueda de un nuevo atractivo para las zonas rurales. Todos los datos serán tratados con los Sistemas Informativos Geográficos (GIS). La investigación confirma que las áreas rurales, a pesar de ser ricas en recursos culturales, medioambientales, históricos, etcétera, todavía no han conseguido explotar estos recursos con fines turísticos, como bien podrían hacer. No obstante, hay varios fondos que pueden ser utilizados, y en las conclusiones se hacen algunas consideraciones útiles para mejorar el atractivo turístico de estas zonas e intentar frenar el descenso demográfico.
\end{abstract}

Palabras clave

Áreas rurales / Despoblación / Cerdeña / Turismo rural / Áreas internas.

\section{Strategies for territorial development and tourism against the depopulation of rural areas in Sardinia (Italy)}

\begin{abstract}
The depopulation of rural areas is a problem for many European regions, including Sardinia (Italy), where several villages are in danger of disappearing. Different policies are being implemented to strengthen their competitiveness, and this paper aims to highlight the opportunities offered using various strategies. Therefore, the main territorial development projects promoted in Sardinia and the role of tourism in the search to make rural areas more attractive will be analysed. All the data will be treated with the Geographic Information Systems (GIS). Research confirms that rural areas, despite being rich in cultural, environmental and historical resources, have been unable to exploit them as well as they could for tourism purposes. However, there are several funds that can be used, and, in the conclusions, some useful considerations are given to make these areas more attractive to tourists and try to halt the demographic decline.
\end{abstract}

\section{Keywords}

Rural areas / Depopulation / Sardinia / Rural tourism / Internal areas.

JEL Codes: 018, 021, R58, R12, L88, L83, J10.

\section{Introducción}

La despoblación de las áreas rurales es un problema que afecta a muchas regiones europeas. Su incidencia puede entenderse mejor si se compara la distribución de la población con la más reciente tipología urbano-rural propuesta por Eurostat (2019), según la cual cada unidad territorial estadística NUTS3 es asignada a una de las siguientes categorías: 1) áreas predominantemente urbanas; 2) áreas intermedias; y 3) áreas predominantemente rurales. A pesar de que las primeras cubren una pequeña

\footnotetext{
*Correspondencia autor: slampreu@uniss.it
} 
superficie (el 12\% de la UE-28), se concentra en ellas alrededor del 40\% de la población total de Europa. Por el contrario, las personas que viven en zonas predominantemente rurales, mucho más extensas que las urbanas, no llegan ni al 30\% del total. Como ha señalado ESPON (2018), las áreas rurales más afectadas por la desertización demográfica en la década 2001-2011 se encuentran principalmente en Bulgaria, Grecia, el sur de Italia y sus islas, el noroeste de España y Portugal. Este es un problema que surge con fuerza también en Cerdeña, donde hay varios pueblos en peligro de desaparecer antes de finales del siglo XXI y donde en los últimos años se han promovido políticas de desarrollo territorial para reforzar la competitividad de las áreas marginales. La mayoría de los proyectos que se están llevando a cabo están dirigidos por los Grupos de Acción Local (GAL) y por diferentes mancomunidades.

Se observa que muchas de las iniciativas se centran en el desarrollo del turismo rural y en la integración entre las diversas actividades de la agricultura y de la artesanía local.

Con este trabajo se intenta llegar a un mayor conocimiento sobre los principales proyectos promovidos en Cerdeña contra la despoblación de las áreas rurales, con el objetivo de valorizarlas en clave turística. El presente estudio pretende, por tanto, subrayar las oportunidades ofrecidas a través de diferentes estrategias para el desarrollo de estas zonas deprimidas.

Con respecto a la estructura del trabajo, el apartado 2 trata el fenómeno de la despoblación de las áreas rurales y destaca cuáles son las principales estrategias de desarrollo adoptadas para combatirlo. Para ello, se realizará, en primer lugar, una revisión de la literatura sobre el tema de la desertización demográfica, y se resaltará la importancia que se concede al turismo rural como elemento potencialmente útil con el fin de reorientar las dinámicas de desarrollo hacia nuevas trayectorias y dimensiones, sobre todo en zonas que se caracterizan por tener un tejido empresarial débil y excesivamente fragmentado. En el apartado 3 se presenta la metodología utilizada y las fuentes de datos, mientras que en el 4 se ofrecen los resultados conseguidos. Por último, en el apartado 5 se hace balance de las políticas llevadas a cabo en Cerdeña para la definición de nuevos escenarios de desarrollo territorial. La investigación confirma que las zonas rurales, a pesar de ser ricas en recursos culturales, medioambientales, históricos, etcétera, todavía no han conseguido explotarlos con fines turísticos, como bien podrían hacer. No obstante, existen numerosos fondos que pueden ser utilizados para esos propósitos, y en las conclusiones se hacen algunas consideraciones útiles para mejorar el atractivo turístico de estas zonas e intentar frenar el descenso demográfico.

\section{Las áreas rurales: entre la despoblación y las nuevas perspectivas de desarrollo}

La despoblación de las áreas marginales es un fenómeno multidimensional y antiguo, que se ha intensificado especialmente desde la segunda mitad del siglo XX (Pazzagli, 2017). Se alimenta, actualmente, de algunas causas como la baja tasa de natalidad o los altos índices de envejecimiento que, combinados entre sí, marcan el destino de territorios que, a veces, son incapaces de ofrecer respuestas eficaces e inmediatas a los desafíos de una economía profundamente cambiante tras la desindustrialización, la globalización y el aumento de la movilidad de la población (Del Romero Renau y Valera Lozano, 2015; European Network for Rural Development [ENRD], 2018; European Parliament, 2008; Muenz, 2007; Reckien y Martínez-Fernández, 2011; Syssner, 2015). El progresivo abandono de los asentamientos rurales es objeto de análisis por disciplinas diferentes, desde la geografía a la sociología, desde la demografía a las ciencias económicas, y se ha acompañado a lo largo de los años de un léxico que ha expresado su gravedad: débâcle o catástrofe demográfica, extinción, etnocidio o "demotanasia", neologismo que combina eficazmente los términos demografía y eutanasia (González Martín, 2018; Pazo y Moragón, 2017).

El descenso de la población se traduce, por tanto, en una situación de absoluta emergencia, que se ha visto agravada por las recientes previsiones de Eurostat y de la OCDE, que para las próximas décadas prevén un mayor crecimiento de las ciudades en detrimento de las zonas rurales, que pueden seguir manteniéndose en un estadio de shrinkage (ESPON, 2017a, 2017b, 2020). Entre los principales 
problemas relacionados con la despoblación podemos citar, entre otros, la erosión del capital identitario; la pérdida de tradiciones, conocimientos y culturas locales; o las amenazas a la biodiversidad (Battino y Lampreu, 2019; ESPON, 2018; Macchi Janica y Palumbo, 2019).

Entre las principales iniciativas adoptadas para reforzar el tejido socioeconómico de las zonas rurales, con el objetivo de frenar el declive demográfico, se encuentran aquellas relacionadas con el desarrollo rural (DR), que durante el período 2014-2020 constituye el segundo pilar de la Política Agrícola Común (PAC). Dentro de este último, puede señalarse el enfoque LEADER (Liaison Entre Actions de Développment de l'Économie Rurale), introducido por primera vez durante el período de programación 1989-1993 y consolidado a lo largo de los años (Cejudo García, Navarro Valverde y Camacho Ballesta, 2018). Este enfoque es un instrumento imprescindible, a través del cual los GAL fomentan la multifuncionalidad y la diversificación en la agricultura, promueven el turismo rural y la participación desde abajo con el actual Community Led Local Development (CLLD) (European Commision, 2012). Además de las políticas de desarrollo rural, existen otras estrategias nacionales y/o regionales explícitamente concebidas para luchar contra la despoblación de las zonas marginales a través de medidas de recuperación social y económica. En Italia, por ejemplo, se está experimentando la Estrategia Nacional para las Áreas Internas (SNAI), que se aplica en 72 áreas-proyecto seleccionadas por todo el país (Figura 1).

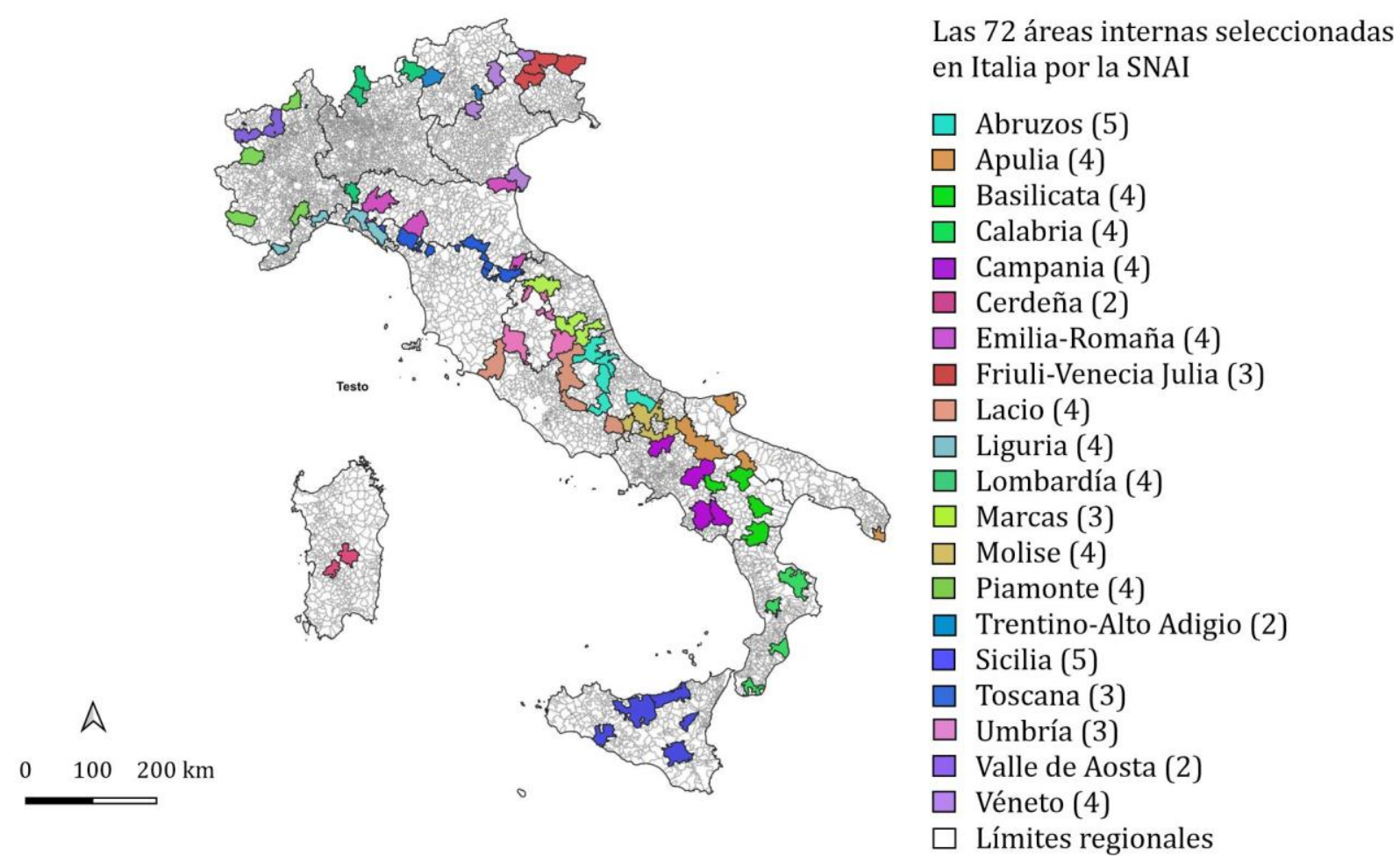

Figura 1. Mapa de las 72 áreas internas seleccionadas en las regiones italianas por la SNAI. Fuente: reelaboración propia con el programa QGIS a partir de datos de la Agencia de Cohesión Territorial.

En este sentido, en los últimos años se ha desarrollado un amplio debate en Italia en torno al concepto de "área interna", término con el que se designan algunas zonas a menudo débiles y afectadas por la despoblación. Las áreas internas se identifican sobre la base de un conjunto definido de indicadores demográficos, socioeconómicos y de distancia de los centros de "servicios esenciales" (llamados polos) como educación, sanidad y transporte (Lucatelli, 2016). Desde el año 2012, en estas zonas actúa la SNAI con el objeto de reforzar la inclusión social y crear más oportunidades de empleo a partir de la valorización del capital territorial escasamente utilizado (Barca, 2012; Barca y Lucatelli, 2014; Cavuta y Ferrari, 2018; Coronato, 2016; Lucatelli, 2015; Scrofani, Novembre y Petino, 2019). 
Las características fundamentales de las áreas internas pueden resumirse en los siguientes puntos: 1) están significativamente alejadas de los polos previamente identificados; 2) guardan importantes recursos medioambientales -recursos hídricos, sistemas agrícolas, bosques, paisajes naturales y humanos- y culturales -bienes arqueológicos, asentamientos históricos, abadías o pequeños museos, entre otros-; y 3) constituyen un territorio muy diversificado como resultado de seculares procesos de antropización y de dinámicas que se han llevado a cabo en los diversos sistemas naturales (Barca, 2013; Lucatelli, 2016).

En cuanto a la palabra "interna" y a la condición de "internalidad", esta debe entenderse especialmente como una forma de perifericidad no solo física sino también relacional y funcional (Prezioso, 2018). Aunque es cierto que el factor distancia puede representar una condición perjudicial, es igualmente cierto que, en virtud de la peculiar localización, se podrían generar respuestas endógenas capaces de dar vida a formas de desarrollo local basadas en la protección y disfrute del paisaje, en la green economy y en la preservación de la identidad territorial (Prezioso, 2018; Scanu et al., 2018).

Los proyectos realizados, ya sea el DR o la SNAI, en la mayoría de los casos tienden, por una parte, a mejorar el acceso de los ciudadanos rurales a los servicios esenciales $\mathrm{y}$, por otra, a favorecer la reinterpretación en clave turística de su capital territorial, la cual podría constituir una importante fuente de ventaja competitiva (Evangelista, Di Matteo y Ferrari, 2018; Meini, 2017).

A pesar de que el concepto de área rural no coincide exactamente con el de área interna, los dos tipos en Italia se prestan fácilmente a solapamientos y a ser tratados conjuntamente dentro de los documentos de política regional. Las áreas rurales e internas se configuran, por tanto, como espacios de enorme potencial inexplorado donde, a las notas críticas, cabe añadir una elevada y potencial calidad de vida, la mayor parte de las veces modelada por las preservadas especificidades geográficas, ambientales, identitarias, culturales o paisajísticas, entre otras.

La Declaración de Cork 2.0 - Una vida mejor en el medio rural (Unión Europea, 2016) reconoce, precisamente, a los territorios marginales estas cualidades, y subraya que las zonas rurales pueden resultar más atractivas para nuevos actores económicos y sociales, respetando las identidades y las vocaciones productivas que deben reinterpretarse a la luz de los principios de la economía circular, de la sostenibilidad medioambiental o de la revisión turística del patrimonio cultural gestionado por las comunidades locales.

Se espera lograr un reposicionamiento competitivo, siempre que se aprovechen las oportunidades ofrecidas por las TIC, cuyo uso en las zonas rurales e internas será decisivo para el fortalecimiento de las relaciones en forma sistémica (Balletto, Milesi, Ladu y Borruso, 2020) con el fin de facilitar la apertura de estas áreas hacia el exterior y para aplicar la más reciente estrategia europea de los Smart Villages lanzada oficialmente en el año 2017 con el Cork 2.0 Action Plan (ENRD, 2018; Pérez-delHoyo y Mora, 2019; Visvizi y Lytras, 2018; Visvizi, Lytras y Mudri, 2019).

La integración entre los sectores productivos tradicionales, las comunidades locales, las TIC y las formas de turismo "alternativo" centradas en el disfrute de los recursos territoriales podría desembocar en nuevos escenarios de desarrollo para zonas consideradas a largo plazo poco atractivas, y que cada vez con más frecuencia acogen una emergente neorruralidad (Di Matteo, 2018; Di Renzo, 2013).

En estas áreas, entonces, desempeña un papel fundamental el turismo rural. Este, para la Organización Mundial del Turismo, se compone de cinco elementos: rural communities, rural areas, rural heritage and culture, rural life y rural activities (Akay, 2020; UNWTO, 2004). Aunque no existe una definición compartida de turismo rural en la literatura, se cree que, sobre todo en la forma 4.0 (turismo interactivo, conectado, social, inteligente y experiencial), podrá ser capaz de estimular sinergias positivas entre actores locales, recursos culturales o actividades tradicionales de agricultura y artesanía, por citar solo algunas de ellas. Entre los elementos que lo caracterizan se pueden señalar, entre otros, el aspecto experiencial, la búsqueda de contacto con la naturaleza y con las poblaciones locales, el interés por la gastronomía tradicional o la atracción por los caminos e itinerarios culturales (Barke, 2004; Cabaleiro Besada, Araújo Vila y Fraiz Brea, 2019; Cawley y Gillmor, 2008; Garau, 2015; Scanu, Madau y Ugolini, 2006; Scanu y Podda, 2016). 
Como han señalado varios autores (Adeyinka-Ojo, Khoo-Lattimorea y Naira, 2014; Gao y Wu, 2017; Xue, Kerstetter y Hunt, 2017), las repercusiones económicas y sociales del turismo en las zonas rurales han sido ampliamente debatidas, llegando cada vez más a ser considerado este sector como un motor para el desarrollo de las áreas marginales (Brandano y Mastrangioli, 2020; Podda, Camerada y Lampreu, 2016). Si es capaz de crear nuevos empleos o de revalorizar los existentes, de favorecer el redescubrimiento, la conexión en red y la integración de los recursos territoriales, así como la preservación del capital natural y cultural, el turismo rural podría ser un instrumento útil contra la despoblación de los núcleos pequeños, y por ello es fuertemente alentado por diversas estrategias llevadas a cabo a escala regional y local.

\section{Metodología y datos}

El presente trabajo, a partir de un análisis de la literatura científica que ha abordado la cuestión de la despoblación en los territorios marginales, junto con la búsqueda de soluciones basadas en la valorización en clave turística de sus recursos culturales, medioambientales y paisajísticos, se centra en el examen de las estrategias de desarrollo promovidas en Cerdeña para frenar la desertización demográfica en las zonas rurales e internas de la isla. En particular, se tendrán en cuenta los principales instrumentos y las políticas regionales aplicadas durante el período de programación 2014-2020, y se comprobará en qué medida el turismo es considerado un driver en los procesos de relanzamiento de la competitividad y del atractivo de estos territorios.

Tras un análisis de contexto relativo a la despoblación, realizado relacionando los estudios de la Región Autónoma de Cerdeña (RAS) con los datos estadísticos de la población, con la dimensión rural del territorio y con las estrategias de relanzamiento adoptadas, se destacarán algunas fortalezas y puntos críticos de las zonas rurales de Cerdeña que pueden influir en su desarrollo turístico. A continuación, se hará referencia a algunas medidas e iniciativas específicas adoptadas por distintos entes territoriales en el marco de diferentes programas expresamente destinados a la valorización turística de los recursos presentes en las zonas rurales.

Los datos e informaciones cuantitativas y cualitativas utilizadas derivan, sobre todo, de los planes de acción y de desarollo aplicados a través de los GAL y de las mancomunidades; de los censos de agricultura y de población; de los archivos digitales abiertos de la Región Autónoma de Cerdeña; del Instituto Nacional de Estadística (ISTAT); del Ministerio de Políticas Agrícolas, Alimentarias y Forestales; y de la Agencia de Cohesión Territorial.

Todos los datos, abiertos y extraídos en varios formatos (shape file, kml, kmz, Gpx, archivos de texto, tablas en excel, CSV, etcétera), de documentos y sitios web públicos, han sido limpiados y procesados para ser tratados por los Sistemas Informativos Geográficos (GIS), cuyo papel, en este caso, es fundamental para la representación de los hechos analizados y para su tratamiento territorializado y relacionado con diferentes dinámicas (demográficas, productivas, económicas...).

\section{Resultados}

La investigación realizada a partir de estudios y documentos oficiales de la Región Autónoma de Cerdeña, unida al tratamiento de datos procedentes de fuentes locales, regionales y nacionales, ha permitido subrayar lo siguiente:

-Cómo es tratado el tema de la despoblación de las zonas rurales e internas dentro de las políticas integradas de desarrollo territorial.

- Las fortalezas y los puntos críticos de las zonas rurales de Cerdeña en relación con su tejido social y económico. 
-Cómo y en qué medida las políticas de desarrollo territorial contribuyen a la definición de nuevos escenarios para las zonas rurales.

\subsection{La despoblación en Cerdeña y su tratamiento dentro de la planificación territorial}

La despoblación de las zonas rurales es un problema de absoluta actualidad también para Cerdeña, región italiana situada en el centro del Mediterráneo occidental que, con una superficie de $24.090 \mathrm{~km}^{2}$ (Mariotti, 2014), contaba en el censo del año 2011 con una población de 1,64 millones de habitantes (Breschi, 2017), y que, según las previsiones del ISTAT, disminuirá en un 17,6\% antes del año 2050 (Breschi y Esposito, 2017). En cuanto a su distribución, se registra una elevada concentración a lo largo de las costas (Brundu, 2013) donde, por otra parte, se localizan las tres principales zonas urbanas de la isla, que acogen ellas solas más de 700.000 personas residentes: la ciudad metropolitana de Cagliari; Sassari con la red metropolitana del norte de Cerdeña; y la ciudad de Olbia (Figura 2). La mayoría de los 377 municipios de Cerdeña son pequeños (algunos muy pequeños, con unos pocos cientos de habitantes) y sufren un acusado declive demográfico. Si se observan los principales índices de estructura de la región -índice de vejez (I.V.), índice de dependencia (I.D.) e índice de sustitución generacional (I.R.)-, se constata su constante empeoramiento, especialmente en el período 2001-2018 (Tabla 1).

Tabla 1. Principales índices de estructura de la Región Autónoma de Cerdeña en el período 2001-2018

\begin{tabular}{|c|c|c|c|c|c|c|c|c|}
\hline & $\begin{array}{c}\text { I.V. } \\
31 / 12 / 01\end{array}$ & $\begin{array}{c}\text { I.V. } \\
31 / 12 / 11\end{array}$ & $\begin{array}{c}\text { I.V. } \\
31 / 12 / 18\end{array}$ & $\begin{array}{c}\text { I.D. } \\
31 / 12 / 01\end{array}$ & $\begin{array}{c}\text { I.D. } \\
31 / 12 / 11\end{array}$ & $\begin{array}{c}\text { I.D. } \\
31 / 12 / 18\end{array}$ & $\begin{array}{c}\text { I.R. } \\
31 / 12 / 01\end{array}$ & $\begin{array}{c}\text { I.R. } \\
31 / 12 / 11\end{array}$ \\
\hline Cerdeña & 116,1 & 164,6 & 212 & 42,7 & 47,9 & 53,8 & 93,1 & 142,8 \\
\hline
\end{tabular}

Fuente: Tuttitalia e ISTAT.

También hay que tener en cuenta que la baja densidad de población de la isla $\left(68,0\right.$ hab./ $\left.\mathrm{km}^{2}\right)$, unida a la despoblación de las áreas rurales, ha generado una situación de emergencia.

Es desde el período de posguerra, y sobre todo en las últimas décadas, cuando la despoblación ha afectado principalmente a las áreas localizadas geográficamente en el centro de la región (Battino y Lampreu, 2017; Palumbo, 2019; Puggioni, 2016), caracterizadas todas ellas por una marcada ruralidad, como demuestra la zonificación realizada por el Programa de Desarrollo Rural Regional ${ }^{1}$ (PDR 2014-2020).

Este continuo declive demográfico se confirma en el estudio encargado por la Región Autónoma llamado "Municipios en extinción" (RAS, 2013) en el cual, a través de un elaborado sistema de proyecciones estadísticas y del uso de indicadores compuestos como el estado de malestar demográfico (EMD), se llegó a la conclusión de 31 núcleos de población estarán en riesgo de desaparición antes del año 2086, todos ellos con una población inferior a 1.000 habitantes (Cocco, Fenu y Lecis Cocco-Ortu, 2016). A estos 31 núcleos hay que añadir otros 47 (Figura 3), que se han alcanzado con métodos menos estrictos y que se identifican como núcelos "en condiciones actuales y previsibles de malestar de-

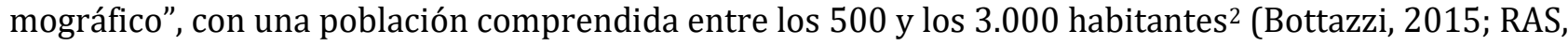
2013).

${ }^{1}$ Esta zonificación está basada en la metodología nacional para perimetrar las áreas rurales en Italia, desarrollada adaptando la utilizada por Eurostat y la OCDE, que identifica en Cerdeña solo un polo urbano que coincide con la ciudad de Cagliari, y clasifica el resto del territorio regional como "rural", aunque con diferentes matices.

2 De los 31 núcleos en riesgo de extinción, 13 tienen menos de 500 habitantes. En cuanto a su localización, 4 se encuentran en las montañas, 26 en las colinas internas, 1 en las colinas costeras y ninguno en las llanuras. El estudio también indica los intervalos de tiempo dentro de los cuales se espera la desertización demográfica: 2 núcelos podrían desaparecer en menos de 20 años, 10 entre 21 y 40 años, 14 entre 41 y 60 años, y 5 después de más de 60 años pero, en cualquier caso, en este siglo. De los otros 47 municipios, 32 pertenecen a áreas internas de la colina, 5 a la colina costera y 10 están situados en las montañas (RAS, 2013). 


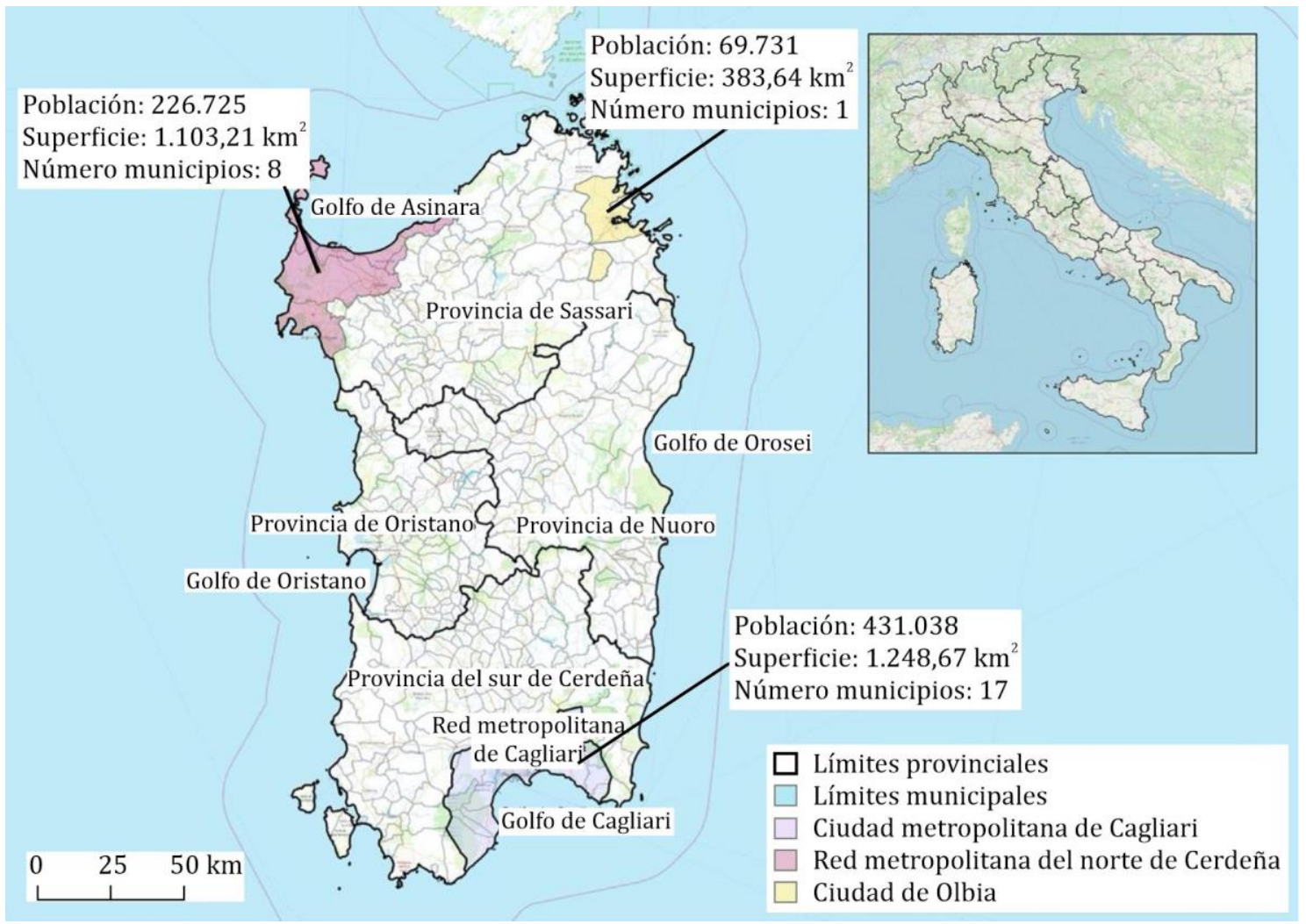

Figura 2. Encuadramiento geográfico de Cerdeña. Fuente: elaboración propia con el programa QGIS a partir de datos del ISTAT y de la RAS).

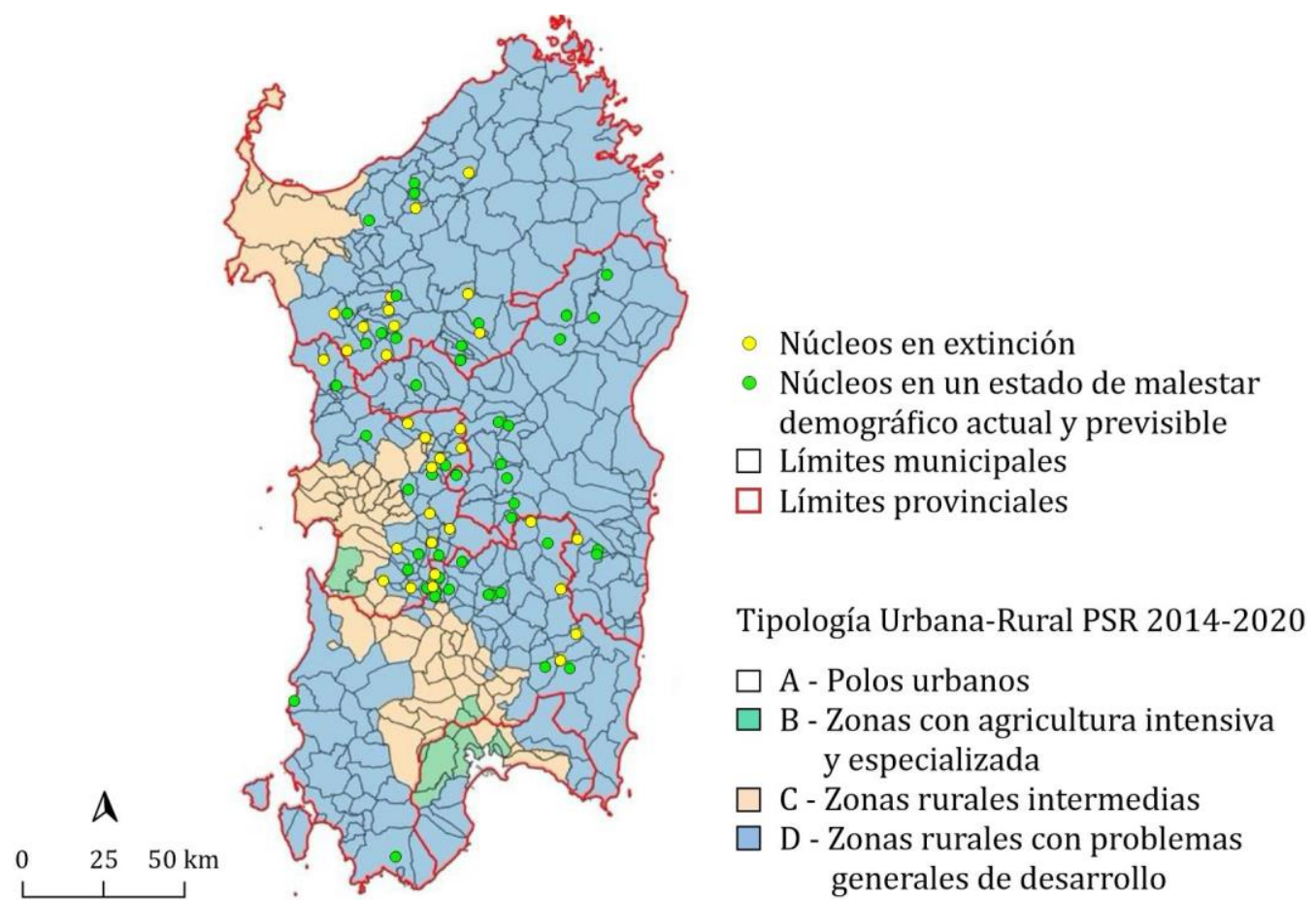

Figura 3. Núcleos en extinción con respecto a la clasificación rural del territorio isleño. Fuente: elaboración propia con el programa QGIS a partir de datos de la RAS y del PDR 2014-2020. 
La Región Autónoma de Cerdeña, para el período de programación 2014-2020, con el fin de satisfacer los objetivos de la Estrategia Europa 2020 y reforzar su competitividad, ha planeado una programación territorial unitaria e integrada. A través de esta última se pretenden superar los desequilibrios territoriales existentes entre las zonas urbanas y rurales, así como entre las zonas centrales y las zonas costeras.

Los principales documentos de política regional adoptados en el actual período son el POR FEDER, que supone 930 millones de euros, y el POR FSE, con un importe de 444,8 millones de euros. Como se observa en el análisis de ambos, el desarrollo territorial en la isla busca integrar los diferentes fondos regionales, nacionales y europeos, con algunas acciones dirigidas específicamente a las zonas urbanas y otras a las áreas rurales e internas (estas dos tipologías son tratadas conjuntamente) (Scanu et al., 2018).

Para las zonas urbanas se recurrirá a las Inversiones Territoriales Integradas (ITI), que se implementarán en los barrios excluidos de las ciudades de Cagliari, Sassari y Olbia ${ }^{3}$, y al Programa Operativo Nacional Ciudad Metropolitana (PON Metro) ${ }^{4}$, que se llevará a cabo en la ciudad metropolitana de Cagliari (Mariotti, Camerada y Lampreu, 2020). Para las zonas rurales se empleará el enfoque CLLD, que se aplicará a través de los $\mathrm{GAL}^{5}$, mientras que para las áreas internas y las mancomunidades serán la SNAI y a la nueva Programación Territorial (PT) ${ }^{6}$ (Figura 4).

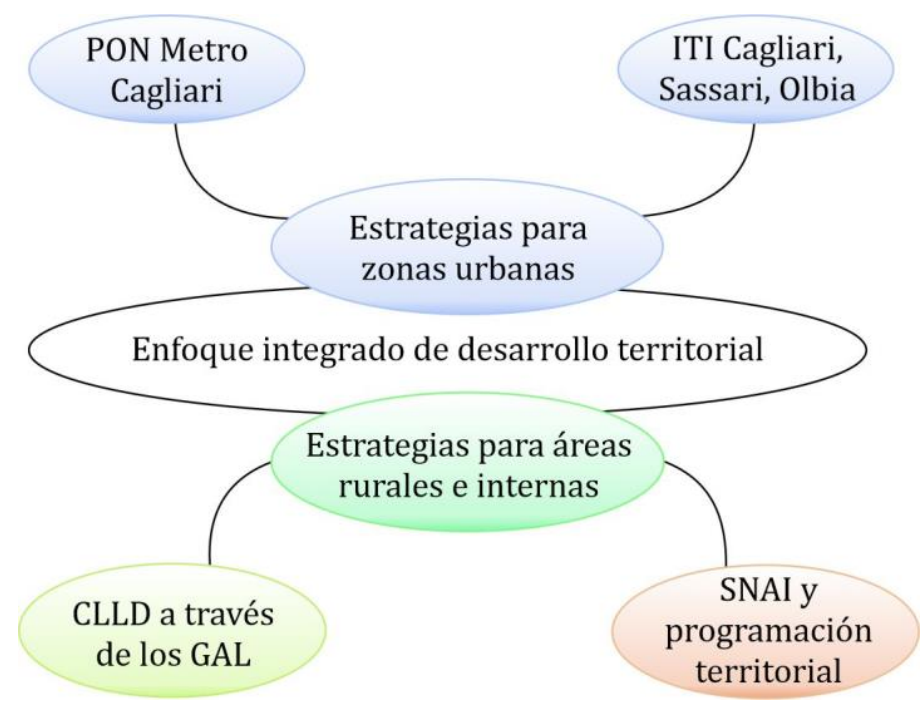

Figura 4. Enfoque integrado de desarrollo regional. Fuente: elaboración propia partir de datos del RAS.

Como demuestra la forma en que se gestiona el desarrollo territorial en las zonas rurales, los dos programas POR FEDER y FSE -que financian ITI, PT y SNAI- se integran perfectamente con el DR, financiado con fondos FEADER. Esto se debe a que la competitividad del sistema regional debe perseguirse de manera estratégicamente unitaria.

En todos los documentos políticos regionales se hace referencia con frecuencia al problema de la despoblación de las zonas rurales y a la necesidad de encontrar soluciones inteligentes, sostenibles e integradoras que puedan favorecer su desarrollo endógeno. El turismo, como subraya también la Es-

\footnotetext{
3 https://www.sardegnaprogrammazione.it/programmazione2014-2020/ITI/

${ }^{4}$ http://www.ponmetro.it/home/ecosistema/viaggio-nei-cantieri-pon-metro/pon-metro-cagliari/

${ }^{5} \mathrm{http://www.regione.sardegna.it/speciali/programmasvilupporurale/}$

${ }^{6}$ https://www.sardegnaprogrammazione.it/programmazione2014-2020/programmazione territoriale/
} 
trategia de Especialización Inteligente de Cerdeña (S3), puede ser un motor para estas zonas que disponen de un enorme capital territorial poco valorizado. Son, precisamente, los recursos locales los que constituyen la base para el nacimiento de formas de turismo rural capaces de conjugar la protección del medio ambiente y de la cultura con su capacidad de crear riqueza y bienestar.

\subsection{Fortalezas y puntos críticos de las zonas rurales de Cerdeña}

Es preferentemente en las zonas rurales donde se realizan las políticas fundamentales de lucha contra la despoblación, con medidas destinadas a valorizar los paisajes, las identidades y las producciones típicas locales, para fomentar su conexión en red y su posible explotación sostenible e inclusiva con fines turísticos (Brundu y Lampreu, 2017).

Los aspectos relacionados con el desarrollo económico en estas áreas son fundamentales, sobre todo teniendo en cuenta que existe en toda Cerdeña un problema de reducida dimensión empresarial (Centro Ricerche Economiche Nord Sud [Crenos], 2019). Esta cuestión se agrava especialmente en los territorios rurales, con pequeñas empresas de carácter familiar, apenas conectadas entre sí e incapaces de explotar economías de escala. Además, al estar poco digitalizadas, especialmente en lo que respecta al sector primario, son también poco competitivas.

La fuerte incidencia del sector agrícola y ganadero en Cerdeña, reflejada en las 60.812 empresas registradas durante el último censo de la agricultura del año 2010 (Figura 5), ha contribuido a la preservación de antiguas tradiciones productivas y a la transmisión de conocimientos técnicos arraigados, expresión de identidad territorial y cuya calidad está confirmada por los productos IGP, DOC y DOP (en el año 2016, según el ISTAT, había 12.369 productores específicamente relacionados con estas marcas en toda Cerdeña, como se puede observar en la Figura 6).

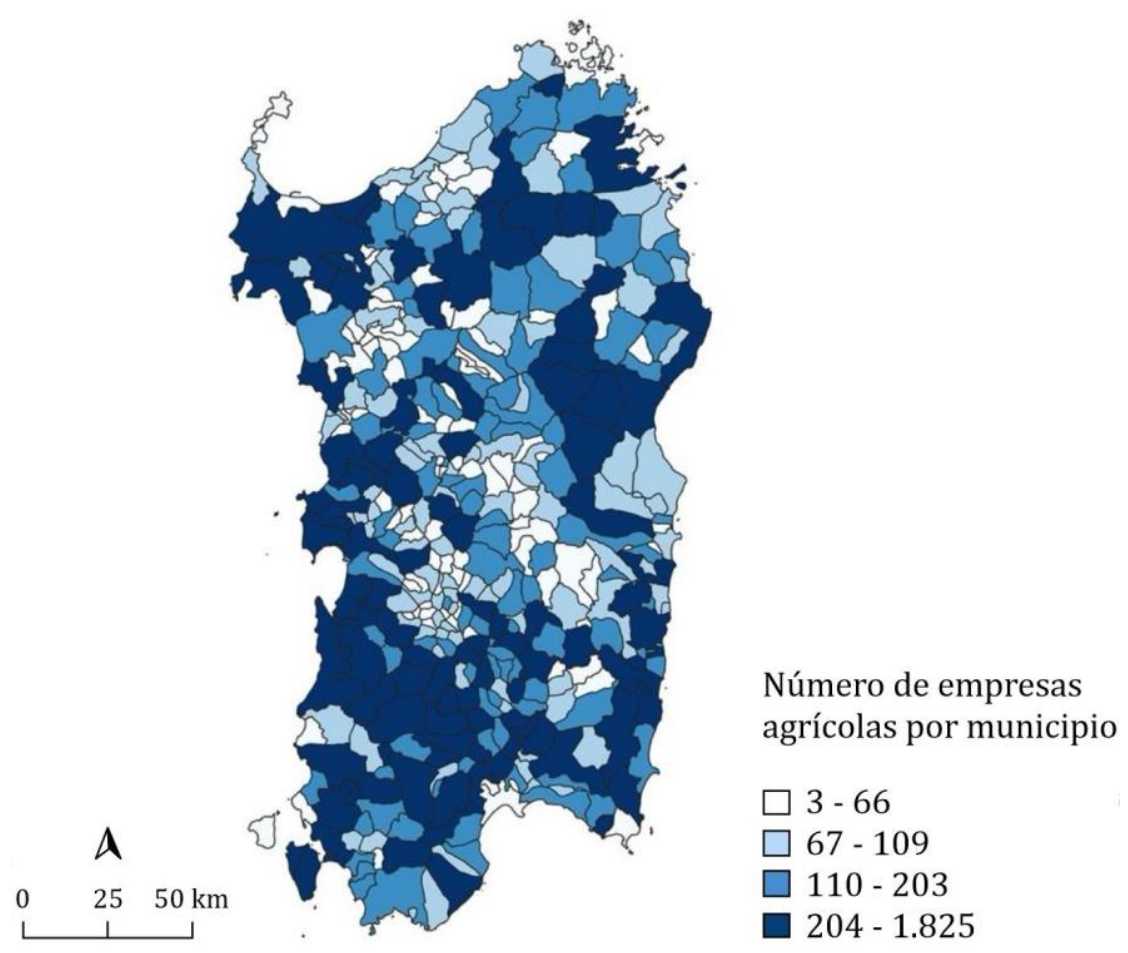

Figura 5. Número de empresas agrícolas por municipio. Fuente: reelaboración del autor con el programa QGIS sobre datos del ISTAT, Censo de la agricultura $2010 \mathrm{y}$ Atlante Statistico dei Comuni. 


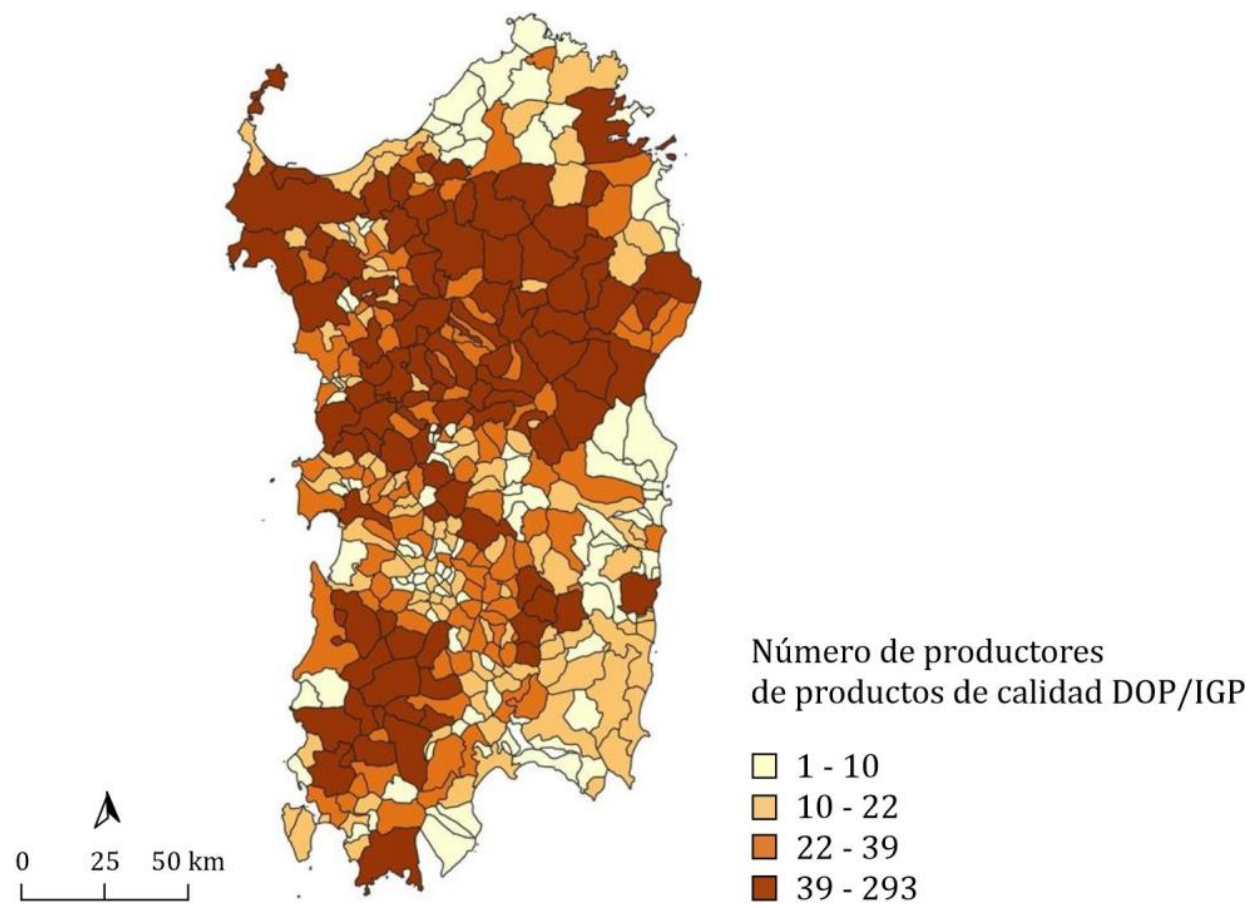

Figura 6. Número de productores de productos DOP/IGP por municipio. Fuente: reelaboración propia con el programa QGIS a partir de datos del ISTAT (2016) y de Atlante Statistico dei Comuni.

Con base en los datos proporcionados por el Ministerio de Políticas Agrícolas, Alimentarias y Forestales (MIPAAF), Cerdeña cuenta con 6 productos con la marca DOP, representados por 3 quesos (Fiore Sardo, Pecorino Sardo y Pecorino Romano), el aceite de oliva virgen extra, el azafrán y la alcachofa espinosa; 2 productos IGP que son el cordero de Cerdeña y los culurgiones d'Ogliastra7; y 2 ETG (Especialidades Tradicionales Garantizadas), que son la mozzarella y la pizza napolitana, cuya producción se encuentra en todas las regiones de Italia.

A estos productos de alta calidad se añaden también los PAT (Productos Agroalimentarios Tradicionales), representados por producciones típicas, elaboradas tradicionalmente desde hace al menos 25 años y debidamente documentadas. Según la relación actualizada por la Región Autónoma ${ }^{8}$, en Cerdeña hay 205 PAT entre bebidas, carnes, condimentos, quesos, pastas frescas, etcétera.

En cuanto al vino, hay que hacer un discurso aparte: en la isla se producen 1 DOCG (Denominación de Origen Controlada y Garantizada), atribuida al Vermentino di Gallura ${ }^{9}$, y se cuentan 17 DOC (Denominación de Origen Controlada) y 15 IGT (Indicación Geográfica Típica).

A estos verdaderos yacimientos alimentarios, cuyo peso es fundamental en la definición del perfil turístico de un destino agroalimentario (Garibaldi, 2017; Miani, 2015), en las zonas rurales de Cerdeña se une la rica presencia de bienes culturales, arqueológicos y de interés histórico (museos, iglesias rurales o abadías, entre otros), además de itinerarios y caminos de la fe y de la trashumancia.

Todo este conjunto de bienes materiales e inmateriales está inmerso en paisajes inalterados debido, en parte, al bajo peso antrópico. Gracias a las especificidades geográficas, los territorios actualmente considerados marginales pueden ser tratados como laboratorios para alojar nuevas soluciones de valorización y desarrollo, en línea con las exigencias de un turismo lento, sostenible y responsable (Battino, Balletto, Borruso y Donato, 2018). A pesar de esto, las áreas rurales de Cerdeña todavía están po-

\footnotetext{
${ }^{7}$ Los culurgiones son un tipo de pasta fresca con relleno de patatas típicos de una subregión de Cerdeña llamada Ogliastra.

8 http://www.regione.sardegna.it/documenti/1_850_20190418163507.pdf

${ }^{9}$ El Vermentino es un tipo de vino blanco originario de la subregión de Cerdeña denominada Gallura.
} 
co equipadas en términos de infraestructuras turísticas, como se puede confirmar especialmente por la escasez de camas y alojamientos ${ }^{10}$ en el interior de la isla. La mayoría de las estructuras se localizan a lo largo de las costas (Figura 7), en función de un turismo aún excesivamente estacional y basado en el modelo sol y playa (RAS, 2018), elemento probado por el hecho de que el $82 \%$ de las presencias turísticas se concentran entre los meses de junio y septiembre ${ }^{11}$ (Crenos, 2019).

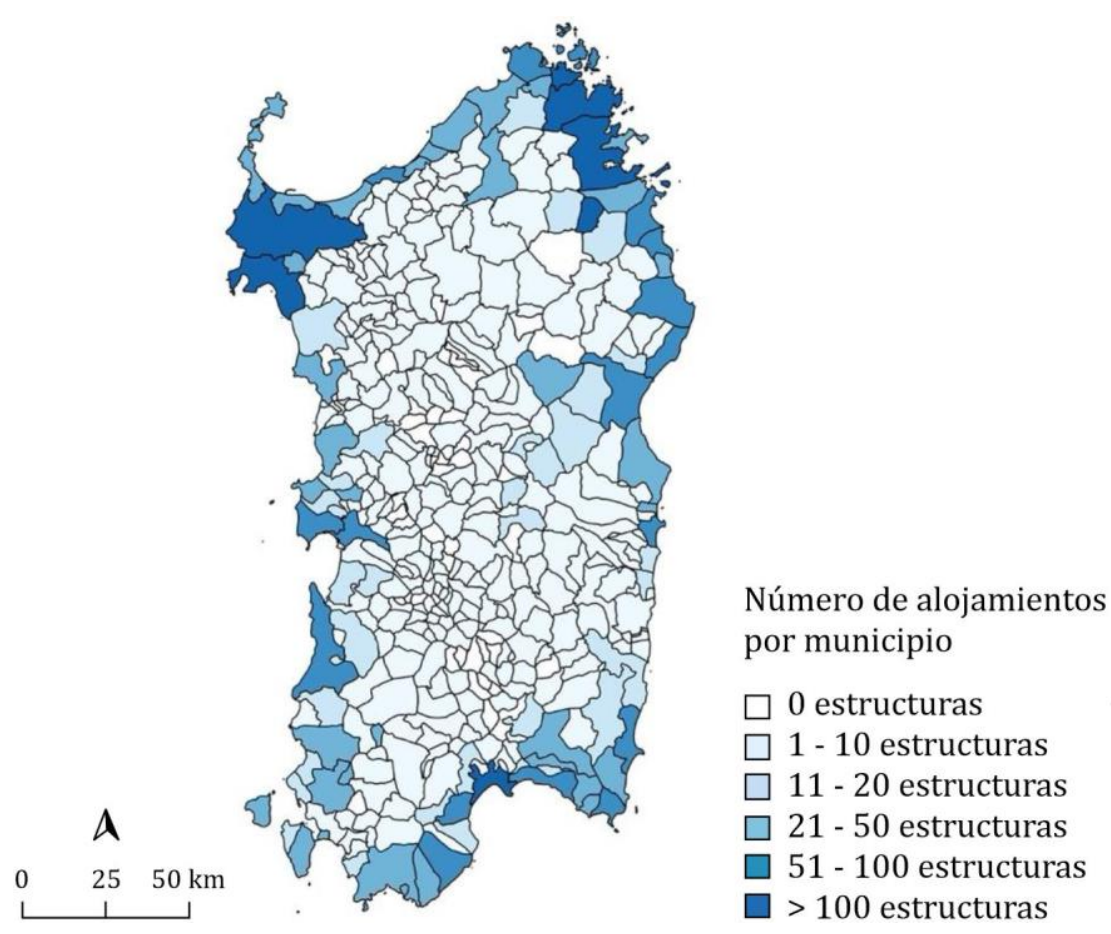

Figura 7. Número de alojamientos por municipio. Fuente: reelaboración propia con el programa QGIS a partir de datos del ISTAT (2016) y del Atlante Statistico dei Comuni.

La distribución de las estructuras responde, pues, a una demanda turística interesada principalmente en un mar cristalino (Ferrari, Laureti y Secondi, 2013) que baña más de $1.800 \mathrm{~km}$ de costa.

Aunque el turismo rural sigue siendo minoritario, en los últimos años se ha producido un aumento de las propuestas de vacaciones y paquetes centrados en el descubrimiento de los territorios menos conocidos. Las actividades más promovidas se refieren a la realización de experiencias al aire libre como senderismo y cicloturismo, visitas a lugares culturales y arqueológicos, degustaciones de vinos y participación en eventos relacionados con las tradiciones, por señalar solo algunos. Son, sobre todo, las personas que viven en las pequeñas comunidades rurales las que proponen nuevas oportunidades de visita, y este elemento indica que el turismo sostenible es aceptado y bien visto como un sector en el que poder invertir. Precisamente por esta razón son diferentes los proyectos de desarrollo territorial que le dedican un amplio espacio, todos dirigidos por los GAL y las mancomunidades.

\footnotetext{
10 Como se observa de las encuestas de ISTAT, en el año 2016 había 4.787 alojamientos (hotel, B\&B y otros) distribuidos de manera desigual en Cerdeña: había 52 municipios completamente desprovistos de alojamiento, otros 52 tenían solo una estructura, 47 municipios contaban con la presencia de 2 estructuras, 38 municipios registraban 3 estructuras, en 32 municipios se localizaban 4 estructuras, 19 municipios contabilizaban 5 estructuras, 56 municipios tenían entre 6 y 10 estructuras, en 31 municipios las estructuras de las que disponían estaban comprendidas entre 11 y 20, y por último 50 municipios contabilizaban más de 20 instalaciones, de las que solo 6 de ellos contaban con más de 100 establecimientos.

11 En el año 2019 se registraron en la región 3.557.557 llegadas y 15.824.985 presencias turísticas.
} 


\subsection{La contribución de las políticas de desarrollo territorial a la definición de nuevos escenarios para las áreas marginales}

Como se ha señalado con anterioridad, las áreas marginales pretenden aumentar su competitividad a través de dos medidas principales:

-Una de ellas se localiza en el marco del DR, regulado por el PDR 2014-2020, y se aplica según indica la medida 19 (Apoyo al desarrollo local LEADER) de los GAL, que disponen de recursos por valor de 93,6 millones de euros.

- La segunda medida, con un valor de casi 370 millones de euros, sería la SNAI junto con la PT, que se ejecuta por las mancomunidades ${ }^{12}$, las cuales, bajo la dirección del Centro Regional de Programación, presentan proyectos específicos de desarrollo local.

Por lo que se refiere a los GAL de Cerdeña, que son agrupaciones que representan tanto a la población rural (a través de los entes públicos: municipios, provincias y comunidades de montaña) como a las organizaciones de emprendedores del territorio, en este período de programación fueron identificados 17 (Figura 8), y cada uno de ellos se encarga de aplicar el enfoque LEADER. Como se refleja en la página web dedicada al DR en Cerdeña, los municipios que forman parte de los GAL son 282 de un total de 377, con una población de alrededor de 550.000 habitantes y con una extensión del 69\% de toda la superficie regional.

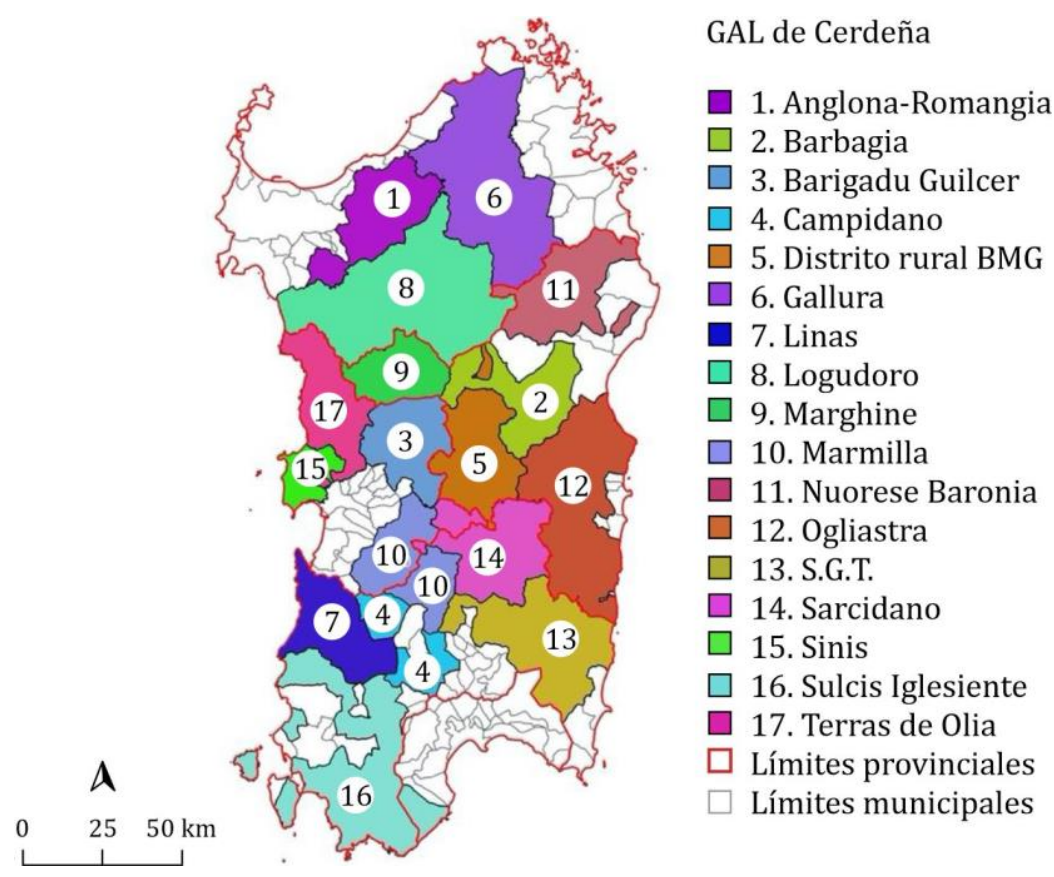

Figura 8. GAL de Cerdeña (2014-2020). Fuente: reelaboración propia con el programa QGIS realizada a partir de datos del PSR y del RAS.

Teniendo en cuenta todas las estrategias de los GAL, reguladas por los Planes de Acción (PdA) elaborados con un enfoque participativo (CLLD), se observa que pretenden concentrarse en los siguientes ámbitos temáticos:

\footnotetext{
12 Las mancomunidades son agregaciones de municipios gobernadas por la L.R. 4 de febrero de 2016, n. 2 “Reorganización del sistema de autonomías locales de Cerdeña", que se encargan de realizar algunas funciones compartidas (transporte local, recogida de residuos o servicios de comedor escolar, entre otras). Sus límites coinciden a menudo con los de las subregiones histórico-geográficas de la isla.
} 
- Desarrollo e innovación de los sectores y sistemas productivos locales (44\%).

-Turismo sostenible (43\%).

-Valorización de bienes culturales y patrimonio artístico (5\%).

- Desarrollo del sector de energías renovables (3\%).

-Inclusión social de grupos específicos desfavorecidos y/o marginales (3\%).

- Calidad de vida y hospitalidad (2\%).

Precisamente, del análisis de todos los PdA se concluye que 11 de ellos han decidido invertir total o parcialmente en el eje del turismo sostenible, proponiendo acciones diversificadas que afectan a los sectores de la receptividad, de la regeneración de las arquitecturas rurales, de las producciones agroalimentarias, de la realización de itinerarios, del marketing territorial y digital, etcétera. A estas intervenciones se añaden las de los otros ámbitos (energías renovables, calidad de vida, etc.) que, al estar interrelacionados entre sí, son capaces de generar externalidades positivas también en el sector del turismo rural.

Por ejemplo, algunos GAL, como el GAL Barbagia, prevén fomentar la creación de una red de productores agroalimentarios con el fin de promover y comercializar sus bienes en el mercado regional, financiando intervenciones estructurales y actividades de formación. De la misma manera, se pretende fomentar la creación de redes entre artesanos y diseñadores mediante inversiones en nuevas tecnologías y actividades de marketing en favor de las empresas ${ }^{13}$.

Otros GAL, como el Barigadu Guilcer, han concentrado su estrategia sobre todo en dos frentes: por una parte, la valorización turística de los atractivos naturales y culturales, con la realización de itinerarios y caminos para el turismo lento, y por otra parte, la promoción de las líneas productivas locales vinculadas a la agricultura y a la artesanía de forma integrada ${ }^{14}$.

Hay otros, como Anglona-Romangia, que aspiran explícitamente a un aumento de las plazas (B\&B y casas rurales) mediante la concesión de subvenciones a fondo perdido a los operadores locales, reforzando también las estrategias de comunicación y de marketing territorial ${ }^{15}$.

Resulta especialmente interesante la acción llevada a cabo por el GAL Ogliastra, que pretende afirmarse como un producto turístico organizado capaz de ofrecer, junto a los atractivos naturales, culturales e históricos, todos los servicios necesarios para facilitar el disfrute por parte de los visitantes ${ }^{16}$.

En los mismos territorios, a los proyectos de desarrollo local promovidos por los GAL se suman los realizados en el marco de la PT, donde se ve el protagonismo de las mancomunidades, individualmente o asociadas entre sí, llamadas a proponer a la Región Autónoma proyectos de desarrollo territorial. Estos proyectos, en la mayor parte de los casos, tienen el objetivo de realizar intervenciones hacia una valorización turística de las áreas interesadas. Los planes de la PT (tanto los aprobados como los pendientes de aprobación) implican casi todo el territorio regional y se integran perfectamente con la SNAI que, en Cerdeña, se desarrolla en dos áreas internas seleccionadas para la experimentación nacional: la mancomunidad Alta Marmilla (15 millones de euros asignados) y la Comunidad de Montaña Gennargentu-Mandrolisai (9 millones de euros asignados) (Figura 9).

Entre todos los proyectos presentados, y a título de ejemplo, se citan tres: el Plan de Desarrollo Territorial (PST) Sarcidano Barbagia, el PST Su Suercone y el PST Marghine.

El PST "Sarcidano Barbagia di Seulo, Tierra de Agua y Piedra", propuesto por 16 municipios de la provincia del Sur de Cerdeña pertenecientes a la mancomunidad Sarcidano-Barbagia di Seulo, a través de cuatro orientaciones estratégicas y seis acciones temáticas, pretende realizar un sistema de recorridos que pueda dar forma a un destino unitario capaz de valorizar las potencialidades del atractivo del Trenino Verde17. En detalle, las seis acciones temáticas se refieren a: 1) la valorización de la oferta

\footnotetext{
13 http://www.galbarbagia.it

14 www.galbarigaduguilcer.it

15 http://www.galanglonaromangia.it

16 http://www.galogliastra.it

17 El Trenino Verde es un tren de camino lento, con meras funciones turísticas, que cruza numerosas zonas rurales de Cerdeña con un gran valor paisajístico.
} 
medioambiental; 2) la puesta en red del Trenino Verde con los otros atractivos territoriales; 3) la mejora de la calidad de vida; 4) la mejora de los servicios esenciales para las poblaciones; 5) la governance territorial18; y 6) la valorización de la oferta arqueológica, histórico-cultural y religiosa.

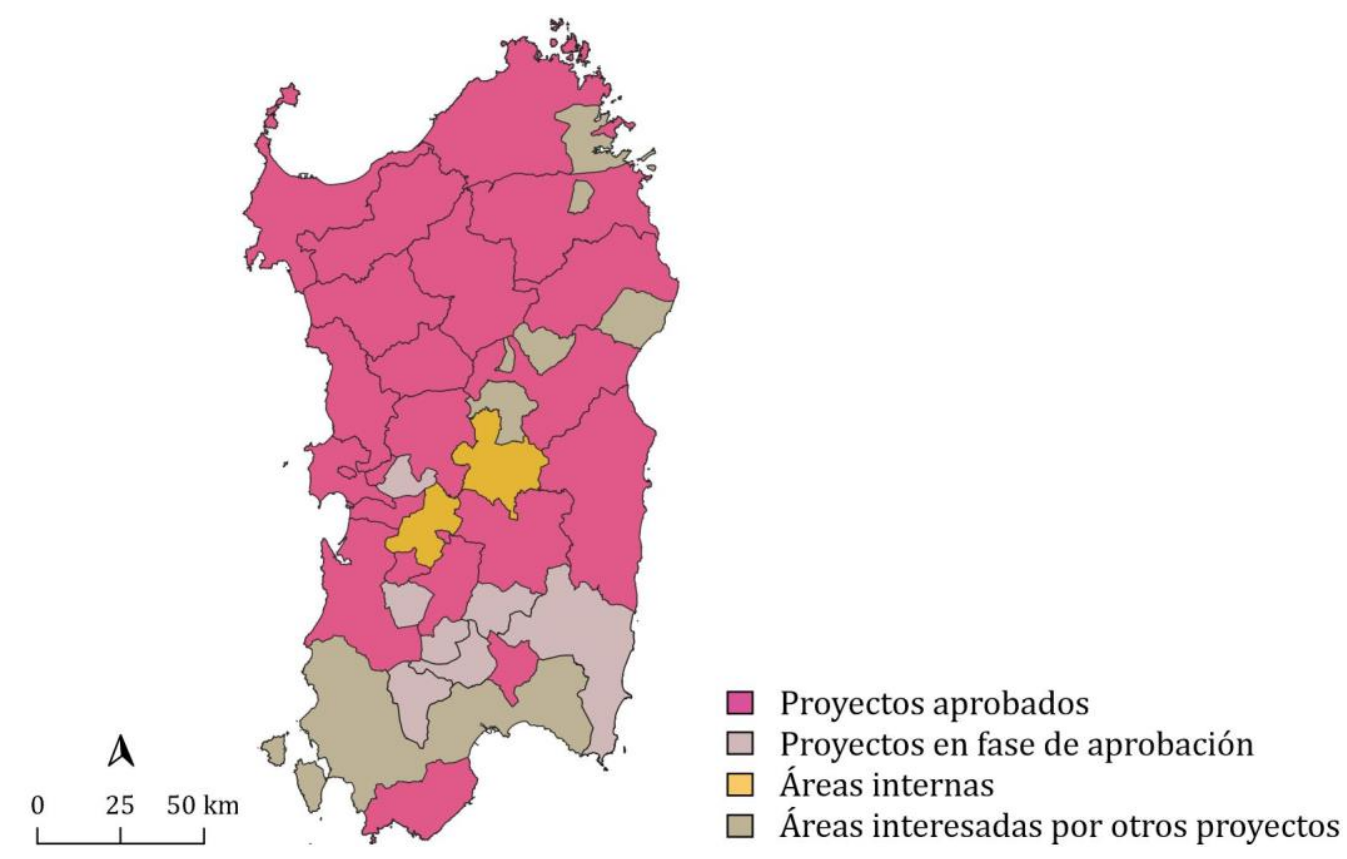

Figura 9. Proyectos de la PT actuados en las mancomunidades de Cerdeña. Fuente: reelaboración del autor con el programa QGIS basada en los datos de www.sardegnaprogrammazione.it

También el PST "Su Suercone, Ambiente Identitario", propuesto por 8 municipios de la provincia de Nuoro pertenecientes a la mancomunidad Nuorese, Gennargentu, Supramonte, Barbagia, aspira a mejorar el atractivo del territorio mediante la creación de un sistema integrado destinado a reconvertir la zona en destino turístico unitario, con especial referencia a los segmentos del turismo medioambiental, excursionista y cultural. Para ello se desarrollan tres acciones temáticas: 1) "Su Suercone entre costa e interno", que realiza intervenciones estructurales de mejora de la accesibilidad; 2) valorización de los atractivos culturales e identitarios, entre los cuales podemos citar museos, sitios arqueológicos y fiestas populares; y 3) governance territorial ${ }^{19}$.

Asimismo, el proyecto "Marghine al Centro - Turismo, Deporte, Cultura y Naturaleza", presentado por 10 municipios de la provincia de Nuoro agregados en la mancomunidad del Marghine, busca reforzar la identidad local mediante la construcción de una oferta turística territorial que integre la promoción y la protección del medio ambiente con la calidad de vida. También en este caso se distinguen tres acciones temáticas: 1) valorización turística de la oferta medioambiental y cultural; 2) mejora de los servicios esenciales para el territorio; y 3) governance territorial 20.

El procedimiento de financiación de los proyectos de desarrollo a través de la PT reserva un papel importante a los actores locales, que son determinantes en la definición de las estrategias y en las operaciones de diseño e inclusión de las diferentes fuerzas económicas y sociales del territorio. Actualmente, hay 37 mancomunidades interesadas por la PT, que han dado vida a 26 agregaciones territoriales, implicando en total a 295 municipios. Los proyectos aprobados son 20 y tienen una cobertura de 343,5 millones de euros.

18 http://www.sardegnaprogrammazione.it/documenti/35_633 20190222125130.pdf

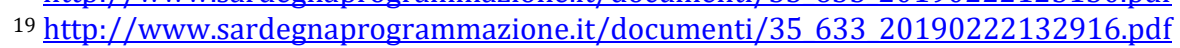

${ }^{20}$ http://www.sardegnaprogrammazione.it/documenti/35 84 20171204114017.pdf 


\section{Conclusiones}

El análisis realizado pone en evidencia el compromiso de la Región Autónoma de Cerdeña con la lucha contra la despoblación de las áreas marginales a través de la promoción y la financiación de medidas que caen en el ámbito de una estrategia unitaria e integrada. De hecho, las políticas para el DR, la PT y la SNAI convergen hacia la activación de nuevas sinergias con el objetivo de realizar un desarrollo endógeno y participativo, frenar la marginalidad y, por tanto, las caídas demográficas.

Entre los sectores en los que se hace especial hincapié, como se ha dicho, figura el turismo y la valorización del patrimonio territorial. Si se gestiona de forma adecuada, el turismo podría contribuir a preservar y transmitir el capital cultural y natural, respetando los principios de la sostenibilidad (Balletto, Milesi, Ladu y Borruso, 2020; Belliggiano y Labianca, 2018; Cerutti, 2018; De Rubertis, Belliggiano y Labianca, 2018; Scanu y Podda, 2018).

Para alcanzar estos objetivos se han puesto en marcha importantes recursos económicos y programas de desarrollo aún en curso de ejecución. Por ello, será interesante comprobar en el futuro si se han conseguido los resultados previstos, midiendo de alguna manera los cambios realizados.

Por ejemplo, para medir la evolución demográfica de los núcleos pequeños se podrían controlar las inscripciones en los registros municipales, las variaciones intercensales de la población y su estructura en función de la composición.

Para evaluar la mejora económica y turística podría ser útil analizar la evolución de algunos indicadores a escala local como, entre otros, las variaciones del índice de turismo por cada municipio, el aumento o la disminución del número de alojamientos, la apertura de nuevas actividades relacionadas con formas específicas de turismo activo o cultural, la creación de consorcios y brands territoriales, la posible integración en red de nuevas estructuras museísticas y lugares de la cultura, la regeneración de caminos y vías pecuarias, etcétera.

Aunque el turismo parece ser un motor para el crecimiento de las zonas rurales de muchas regiones (Randelli y Martellozzo, 2019), es necesario, en cualquier caso, que se desarrolle coherentemente con la identidad de los territorios. Por tanto, el sector debe mostrarse capaz de crear valor a partir de la restitución de centralidad a las producciones típicas locales y al patrimonio presente en las poblaciones, con sus tradiciones y sus valores, pero al mismo tiempo las comunidades tienen que ser protagonistas de los procesos de cambio.

También por ello resulta fundamental la capacidad de conseguir aprovechar las oportunidades ofrecidas desde las nuevas tecnologías para emprender recorridos que faciliten el tránisto de los territorios "periféricos" de Cerdeña hacia una dimensión inteligente, funcional y catalizadora para nuevos residentes y nuevas profesiones relacionadas, como, por ejemplo, aquellas vinculadas con la economía del conocimiento.

A la luz de todo lo que se ha visto, para invertir la tendencia a la despoblación mediante la promoción turística de las zonas rurales de Cerdeña podrían ser útiles las siguientes medidas:

1) Llevar a cabo los proyectos de valorización turística sostenible e inteligente de los GAL y de las mancomunidades ya financiados y en curso de realización, y evaluar sus efectos y sus impactos.

2) Seguir invirtiendo en formación para las empresas que operan en los sectores del turismo y de las producciones locales.

3) Coordinar a nivel regional políticas de marketing turístico con el fin de valorizar las realidades rurales de manera coherente y fuerte.

4) Incentivar la creación de alojamientos extrahoteleros como bnb, casas rurales, hostales, etcétera, especialmente en las zonas rurales, y acompañarlos en la estructuración de una imagen bien definida. En este caso podría ser útil, también, recuperar las casas deshabitadas de los centros históricos y reconvertirlas para usos turísticos.

5) Reforzar las redes entre empresas ligadas a los sectores de la artesanía, del vino y de la gastronomía con las del turismo, fomentando el diseño y la venta de experiencias para vivir el territorio de una manera original y en contacto con las comunidades. 
6) Promover la seguridad y el uso de caminos y rutas a pie, en bicicleta o a caballo en respuesta a una creciente demanda de turismo lento y sostenible.

El desarrollo turístico en las zonas rurales es posible, incluso en las que sufren despoblación, siempre que las comunidades locales participen directamente en los procesos de valorización del territorio y puedan beneficiarse de una diversificación de las actividades históricamente practicadas. Ciertamente, no se trata de procesos sencillos y rápidos, pues transformaciones de este tipo requieren tiempo e implican numerosos aspectos que tienen que ver con la esfera cultural, social e identitaria de las poblaciones rurales. Sin embargo, la existencia de numerosos y diversificados proyectos de desarrollo local, en cuanto que realizados de manera participativa, indica una voluntad de las comunidades locales de avanzar hacia una regeneración turística sostenible de sus territorios.

El universo rural, por tanto, puede ser un espacio de oportunidades y de vida, capaz de garantizar, en primer lugar, el pleno disfrute del derecho de ciudadanía y, en segundo lugar, el mantenimiento de una fuerte interconexión con otras realidades y, en consecuencia, un alto grado de apertura hacia el exterior.

Aumentar las oportunidades de empleo relacionadas con el turismo, mejorar la calidad de vida, reforzar las interconexiones y las infraestructuras, la posibilidad de aprovechar el potencial de las tecnologías digitales y de disfrutar de un medio ambiente limpio y sano podrían ser algunos ingredientes para reforzar el atractivo y la competitividad de las zonas rurales. La combinación de estos elementos, si se gestionan de manera eficaz, podría llevar, quizás, a una reducción de los procesos de despoblación que afectan de forma notoria a las zonas marginales.

\section{Bibliografía}

Adeyinka-Ojo S. F., Khoo-Lattimorea, C., y Naira, V. (2014). A framework for rural tourism destination management and marketing organisations. Procedia - Social and Behavioral Sciences, 144(20), 151-163.

DOI: https://doi.org/10.1016/j.sbspro.2014.07.284

Akay, B. (2020). Examining the rural tourism experiences of tourists in emerging rural tourism destination: Burdur province, Turkey. GeoJournal of Tourism and Geosites, 29(2), 534-544.

Recuperado de: http://gtg.webhost.uoradea.ro/PDF/GTG-2-2020/gtg.29212-487.pdf

Balletto, G., Milesi, A., Ladu, M., y Borruso, G. (2020). A dashboard for supporting slow tourism in green infrastructures. A methodological proposal in Sardinia (Italy). Sustainability, 12(9), 1-23. DOI: https://doi.org/10.3390/su12093579

Barca, F. (2012). Metodi ed obiettivi per un uso efficiente dei Fondi comunitari 2014-2020. Roma, Italia: Ministero della Coesione Territoriale.

Recuperado de: https://www.reterurale.it/flex/cm/pages/ServeBLOB.php/L/IT/IDPagina/10538

Barca, F. (2013). Intervento conclusivo al Forum Aree Interne: nuove strategie per la programmazione 2014-2020 della politica di coesione territoriale. Rieti (Italia), 11-12 de marzo de 2013.

Barca, F., y Lucatelli, S. (2014). Sommario. En F. Barca, P. Casavola y S. Lucatelli (Eds.), Strategia Nazionale per le Aree Interne: definizione, obiettivi, strumenti e governance. Recuperado de: http://www.agenziacoesione.gov.it

Barke, M. (2004). Rural tourism in Spain. International Journal of Tourism Research, 6(3), 137-149. DOI: https://doi.org/10.1002/jtr.480

Battino, S., Balletto, G., Borruso, G., y Donato, C. (2018). Internal areas and smart tourism. Promoting territories in Sardinia Island. Internal Conference on Computational Science and Its Applications - ICCSA 2018 (pp. 44-57). DOI: https://doi.org/10.1007/978-3-319-95174-4 4

Battino, S., y Lampreu, S. (2017). Strategie di valorizzazione e promozione in chiave turistica del patrimonio culturale nelle aree interne. Un caso in Sardegna. Annali del Turismo, VI, 83-105. Recuperado de: http://www.geoprogress.eu/wp-content/uploads/2018/03/AdT2017-04_Battino_Lampreu.pdf

Battino S., y Lampreu, S. (2019). The role of the sharing economy for a sustainable and innovative development of rural areas. A case study in Sardinia (Italy). Sustainability, 11(11), 3004, 1-20. DOI: https://doi.org/10.3390/su11113004

Bottazzi, G. (2015). Variabili demografiche e sviluppo locale. Considerazioni sullo spopolamento in Sardegna. En B. Meloni (Ed.), Aree interne e progetti d'area (pp. 77-88). Torino, Italia: Rosenberg\&Seller. 
Brandano, M. G., y Mastrangioli, A. (2020). Quanto è importante il turismo nelle aree interne italiane? Un'analisi sulle aree pilota. EyesReg, 10(1). [Numero Speciale "Nuove sfide per lo sviluppo delle aree interne"].

Recuperado de: https://www.eyesreg.it/2020/quanto-e-importante-il-turismo-nelle-aree-interne-italianeunanalisi-sulle-aree-pilota/

Breschi, M. (2017). La Sardegna e la mobilità del nuovo millennio. En B. Brundu (Ed.), Movimenti di popolazione in Sardegna nel nuovo millennio (pp. 15-32). Udine, Italia: Forum.

Breschi, M., y Esposito, M. (2017). Per una geografia della fecondità in Sardegna. En M. Breschi y E. Cioni (Eds.), Fare figli in Sardegna (pp. 25-42). Udine, Italia: Forum.

Brundu, B. (2013). Il popolamento costiero della Sardegna, tra storia ed economia. En M. Breschi (Ed.), Popolamento e transizione demografica in Sardegna (pp. 47-64). Udine, Italia: Forum.

Brundu, B., y Lampreu, S. (2017). Tra globale e locale: la valorizzazione delle risorse territoriali per il rilancio delle aree interne. En M. Talia (Ed.), Un futuro affidabile per la città. Apertura al cambiamento e rischio accettabile nel governo del territorio (pp. 63-67). Roma-Milano, Italia: Planum.

Cabaleiro Besada, A., Araújo Vila, N., y Fraiz Brea, J. A. (2019). Grado de desarrollo del turismo rural en Galicia: la oferta complementaria como clave del éxito. Revista Galega de Economía, 28(1), 13-30.

DOI: http://dx.doi.org/10.15304/rge.28.1.6188

Cavuta, G., y Ferrari, F. (Eds.). (2018). Turismo e aree interne. Roma, Italia: Aracne.

Cawley, M., y Gillmor, D. A. (2008). Integrated rural tourism: Concepts and practice. Annals of Tourism Research, 35(2), 316-337. DOI: https://doi.org/10.1016/j.annals.2007.07.011

Cejudo García, E., Navarro Valverde, F. A., y Camacho Ballesta, J. A. (Eds.). (2018). Nuevas realidades rurales en tiempos de crisis: territorios, actores, procesos y políticas. Granada: Universidad de Granada.

Cerutti, S. (2018). Una geografia delle progettualità sostenibili nelle Valli dell'Ossola, Piemonte. En G. Cavuta y F. Ferrari (Eds.), Turismo e aree interne (pp. 111-127). Roma, Italia: Aracne.

Cocco, F., Fenu, N., y Lecis Cocco-Ortu, M. (Eds.). (2016). SPOP. Istantanea dello spopolamento in Sardegna. Siracusa, Italia: Letteraventidue.

Coronato, M. (2016). Gli strumenti europei a servizio delle aree interne. Bollettino della Associazione Italiana di Cartografia, 157, 53-59. D0I: https://doi.org/10.13137/2282-572X/13571

Crenos. (2019). Economia della Sardegna. 26ํ Rapporto 2019. Cagliari, Italia: Arkadia. Recuperado de: https://crenos.unica.it/crenosterritorio/sites/default/files/allegati-pubblicazionites/26\%20\%C2\%B0\%20Rapporto\%20CRENoS\%20sull\%27Economia\%20della\%20Sardegna.pdf

De Rubertis, S., Belliggiano, A., y Labianca, M. (2018). Per uno sviluppo territoriale integrato. Il piano strategico del turismo della regione Puglia 2016-2025 e il ruolo dei territori rurali. En M. Prezioso (Ed.), Sostenibilità e responsabilità dello sviluppo (pp. 253-267). Roma, Italia: Aracne.

Del Romero Renau, L., y Valera Lozano, A. (2015). Teruel, territorio en decrecimiento: dinámicas y oportunidades. Ager. Revista de Estudios sobre Despoblación y Desarrollo Rural, 19, 85-116. DOI: https://doi.org/10.4422/ager.2015.09

Di Matteo, D. (2018). Perifericità o neo-ruralità? Evidenze dall'area Casentino-Valtiberina, Toscana. En G. Cavuta y F. Ferrari (Eds.), Turismo e aree interne (pp. 173-197). Roma, Italia: Aracne.

Di Renzo, E. (2013). Neo-ruralità e cibo geografico. Una formula "filosofale" per l'Abruzzo. Documenti Geografici, 2. Recuperado de: https://www.documentigeografici.it/index.php/docugeo/article/view/41

ENRD. (2018). Borghi intelligenti. Nuova linfa per i servizi rurali. Lussemburgo, Lussemburgo: Ufficio delle Pubblicazioni dell'Unione Europea.

Recuperado de: https://enrd.ec.europa.eu/sites/enrd/files/enrd_publications/publi-enrd-rr-26-2018-it.pdf

Esparcia, J., y Noguera, J. (2001). Los espacios rurales en transición. En J. Romero, A. Morales, J. Salom y F. Vera (Eds.), La periferia emergente (pp. 343-372). Barcelona: Ariel.

ESPON. (2017a). Inner Peripheries: National territories facing challenges of access to basic services of general interest. Draft Handbook, version 07/08/2017. Luxembourg, Luxembourg: ESPON EGTC. Recuperado de: https://www.espon.eu/inner-peripheries

ESPON. (2017b). Shrinking rural regions in Europe. Towards smart and innovative approaches to regional development challenges in depopulating rural regions. Luxembourg, Luxembourg: ESPON EGTC. Recuperado de: https ://www.espon.eu/sites/default/files/attachments/ESPON\%20Policy\%20Brief\%20on\%20Shrinking\% 20Rural\%20Regions.pdf

ESPON. (2018). Fighting rural depopulation in Southern Europe. ESPON EGTC, Luxembourg. Recuperado de: https ://www.espon.eu/sites/default/files/attachments/af-espon_spain_02052018-en.pdf

ESPON. (2020). ESCAPE. European shrinking rural areas: Challenges, actions and perspectives for territorial governance. Luxembourg, Luxembourg: ESPON EGTC. Recuperado de: https://www.espon.eu/escape 
Eurostat. (2019). Methodological manual on territorial typologies - 2018 edition. Luxembourg, Luxembourg: Publications Office of the European Union. DOI: http://doi.org/10.2785/930137

European Commission. (2012). Community-led local development, Cohesion Policy 2014-2020. Brussels, Belgium: European Commission.

Recuperado de: http://ec.europa.eu/regional policy/sources/docgener/informat/2014/community en.pdf

European Parliament. (2008). Shrinking regions: A paradigm shift in demography and territorial development. Brussels, Belgium: European Parliament. Recuperado de: https://www.europarl.europa.eu/thinktank/en/document.html?reference=IPOL-REGI_ET(2008)408928

Evangelista, V., Di Matteo, D., y Ferrari, F. (2018). La Strategia Nazionale delle Aree Interne e il turismo: appunti di riflessione. En G. Cavuta y F. Ferrari (Eds.), Turismo e aree interne (pp. 91- 110). Roma, Italia: Aracne.

Ferlaino, F. (2015). Le aree interne. Pre-testo per una postmodernità sostenibile. En B. Meloni (Ed.), Aree Interne e Progetti d'Area (pp. 159-173). Torino, Italia: Resenberg \& Seller.

Ferrari, G., Laureti, T., y Secondi, L. (2013). Un análisis CGE del impacto del turismo en el sistema economico de Cerdeña. En G. Ferrari, J. M. Montero Lorenzo, J. Mondéjar Jiménez y M. Vargas Vargas (Eds.), Investigaciones, métodos y análisis del turismo (pp. 29- 53). Oviedo: Septem.

Gao, J., y Wu, B. (2017). Revitalizing traditional villages through rural tourism: A case study of Yuanjia Village, Shaanxi Province, China. Tourism Management, 63, 223-233.

DOI: http://doi.org/10.1016/j.tourman.2017.04.003

Garau, C. (2015). Perspectives on cultural and sustainable rural tourism in a smart region: The case study of Marmilla in Sardinia (Italy). Sustainability, 7(6), 6412-6434. DOI: http://doi.org/10.3390/su7066412

Garibaldi, R. (2017). In viaggio per cibo e vino. Opportunità per un nuovo turismo integrato. Roma, Italia: Aracne.

González Martín, A. (2018). La demografía es el destino, Laponia nos invade. Documento de Análisis 09/2018. Madrid: Instituto Espanol de Estudios Estratégicos.

Recuperado de:http://www.ieee.es/Galerias/fichero/docs_analisis/2018/DIEEEA09-2018 Laponia_AGM.pdf

Karcagi Kováts, A., y Katona Kovács, J. (2012). Factors of population decline in rural areas and answers given in EU member states' strategies. Studies in Agricultural Economics, 114, 49-56.

Recuperado de: https://studies.hu/wp-content/uploads/2019/05/2198.pdf

Lucatelli, S. (2015). Quali e cosa sono le aree interne. En F. Monaco y W. Tortorella (Eds.), I Comuni della Strategia Nazionale Aree Interne (pp. 17-30). Roma, Italia: IFEL Fondazione ANCI. Recuperado de:

https://www.fondazioneifel.it/documenti-epubblicazioni/item/download/303 49140d30b741b7114e36bf56e049fe8c

Lucatelli, S. (2016). Strategia Nazionale per le Aree Interne: un punto a due anni dal lancio della Strategia. Agriregionieuropa, 45. Recuperado de:

https://agriregionieuropa.univpm.it/it/content/article/31/45/strategia-nazionale-le-aree-interne-unpunto-due-anni-dal-lancio-della

Macchi Janica, G., y Palumbo, A. A. (Eds.). (2019). Territori spezzati, spopolamento e abbandono nelle aree interne dell'Italia contemporanea. Roma, Italia: CISGE.

Mariotti, G. (2014). Analysis on regional vulnerability to rising energy prices: Focus on Sardinia (Italy). Social Sciences, 3 (4-1), 57-60. DOI: http://doi.org/10.11648/j.ss.s.2014030401.16

Mariotti, G., Sechi Nuvole, M., Camerada, M. V., y Carrus, S. (2018). Risorse e servizi di qualità come strumento di competitività turistica. Analisi della performance regionale: un focus sulla Sardegna. En M. Sechi (Ed.), AGEI Geotema, supl. 8, 4-22. Recuperado de:

https://www.ageiweb.it/geotema/wp-content/uploads/2019/10/Suppl2018 2 Mariotti SechiNuvole Camerada Carrus.pdf

Mariotti, G., Camerada, M. V., y Lampreu, S. (2020). Gli Investimenti Territoriali Integrati in ambito urbano. L'esperienza della Sardegna. En M. Talia (Ed.), La città contemporanea: un gigante dai piedi d'argilla. Atti della Conferenza Internazionale. Urbanpromo XVI Edizione Progetti per il Paese, 15 Novembre 2019 (pp. 257-262). Roma-Milano, Italia: Planum. Recuperado de: http://www.planum.net/planum-magazine/planum-publisherpublication/citta-contemporanea-atti-urbanpromo-2019

Meini, M. (2017). Le potenzialità turistiche delle aree interne. Nuova attrattività e rinnovate sfide. En M. Marchetti, S. Panunzi y R. Pazzagli (Eds.), Aree interne. Per una rinascita dei territori rurali e montani (pp. 95-101). Soveria Mannelli, Italia: Rubettino.

Meloni, B. (Ed.). (2015). Aree interne e progetti d'area. Torino, Italia: Resenberg \& Seller.

Miani, F. (2015). Il cibo da eredità delle culture locali ad attrattore del turismo gastronomico. Annali del Turismo, IV, 11-24.

Recuperado de: http://www.geoprogress.eu/wp-content/uploads/2016/07/AdT2015-1-F.-MIANI.pdf 
Muenz, R. (2007). Aging and demographic change in european societies: Main trends and alternative policy options. SP Discussion Paper No. 0703. Washington D.C., WA: The World Bank, Social Protection. Recuperado de: http://documents.worldbank.org/curated/en/589301468329401986/Aging-and-demographic-change-inEuropean-societies-main-trends-and-alternative-policy-options

Muresan, I. C., Oroian, C. F., Harun, R., Arion, F. H., Porutiu, A., Chiciudean, G. O., Todea, A., y Lile. R. (2016). Local residents' attitude toward sustainable rural tourism development. Sustainability, 8(1), 1-14. DOI: https://doi.org/10.3390/su8010100

Noguera, J., y Copus, A. (2016). Inner peripheries: What are they? What policies do they need? Agriregionieuropa, 45. Recuperado de: https://agriregionieuropa.univpm.it/it/content/article/31/45/inner-peripheries-whatare-they-what-policies-do-they-need

Palumbo, A. A. (2019). La gestione associata come strategia per le politiche di riterritorializzazione in Sardegna: enti locali e neoruralità. En G. Macchi Janica y A. A. Palumbo (Eds.). Territori spezzati, spopolamento e abbandono nelle aree interne dell'Italia contemporanea (pp. 227-234). Roma, Italia: CISGE.

Pazo, A. J., Moragón, M. P. (2017). El despoblamiento en Galicia: la visualización de la "catastrofe". Revista de Estudios sobre Despoblación y Desarollo Rural, 123-154. DOI: http://doi.org/10.4422/ager.2018.02

Pazzagli, R. (2017). Un Paese scivolato a valle. Il patrimonio territoriale delle aree interne italiane tra deriva e rinascita. En M. Marchetti, S. Panunzi y R. Pazzagli (Eds.), Aree interne. Per una rinascita dei territori rurali e montani (pp. 17-25). Soveria Mannelli, Italia: Rubettino.

Pérez-delHoyo, R., y Mora, H. (2019). Towards a new sustainable development model for smart villages. En A. Visvizi y M. D. Lytras (Eds.), Smart villages in the EU and beyond (pp. 49-62). Bingley, England: Emerald.

Podda, C., Camerada, M. V., y Lampreu, S. (2016). Cartografia e promozione del turismo in aree a economia debole. Dal marketing territoriale ai percorsi a base culturale. Bollettino dell'Associazione Italiana di Cartografia, 157, 92-104. Recuperado de: https://www.openstarts.units.it/bitstream/10077/14032/1/AIC 157_Podda Camerada Lampreu.pdf

Prezioso, M. (2015). Quali investimenti urbani di breve periodo per un futuro europeo di lunga durata. En R. Cappellin, M. Baravelli, M. Bellandi, R. Camagni, E. Ciciotti y E. Marelli (Eds.), Investimenti, innovazione e città: una nuova politica industriale per la crescita (pp. 389-397). Milano, Italia: EGEA.

Prezioso, M. (2017). Aree interne e loro potenzialità nel panorama italiano e europeo. Introduzione al tema. GEOTEMA, 55, 68-75. Recuperado de: https://www.ageiweb.it/geotema/wp-content/uploads/2020/02/GEOTEMA 55_Prezioso_13.pdf

Prezioso, M. (Ed.). (2018). Quale territorial impact assessment della coesione territoriale nelle regioni italiane. La concettualizzazione del problema. Bologna, Italia: Pàtron.

Puggioni, G. (2016). Fenomeno dello spopolamento. En F. Cocco, N. Fenu y M. Lecis Cocco-Ortu (Eds.), SPOP. Istantanea dello spopolamento in Sardegna (pp. 26-29). Siracusa, Italia: Letteraventidue.

Randelli, F., y Martellozzo, F. (2019). Is rural tourism-induced built-up growth a threat for the sustainability of rural areas? The case study of Tuscany. Land Use Policy, 86, 387-398.

DOI: https://doi.org/10.1016/j.landusepol.2019.05.018

RAS. (2013). Comuni estinzione. Gli scenari dello spopolamento in Sardegna. Progetto IDMS - 2013. Cagliari, Italia: Centro Regionale di Programmazione, Regione Autonoma della Sardegna.

Recuperado de: https://www.sardegnaprogrammazione.it/documenti/35 84 20140120091324.pdf

RAS. (2014). POR FSE Sardegna 2014-2020. Cagliari, Italia: Centro Regionale di Programmazione, Regione Autonoma della Sardegna.

Recuperado de: https://www.sardegnaprogrammazione.it/programmazione2014-2020/POFSE/

RAS. (2015). PDR 2014-2020. Cagliari, Italia: Programma di Svilluppo Rurale, Regione Autonoma della Sardegna. Recuperado de: http://www.regione.sardegna.it/speciali/programmasvilupporurale/psr-20142020/ilprogramma

RAS. (2015). POR FESR Sardegna 2014-2020. Cagliari, Italia: Sardegna Programmazione, Regione Autonoma della Sardegna.

Recuperado de: https://www.sardegnaprogrammazione.it/index.php?xsl=1384\&s=278013\&v=2\&c=12950

RAS. (2018), Destinazione Sardegna 2018-2021, Piano Strategico di Sviluppo e Marketing Turistico della Sardegna. Cagliari, Italia: Regione Autonoma della Sardegna.

Recuperado de: http://www.regione.sardegna.it/documenti/1_231_20181221121007.pdf

Reckien, D., y Martínez-Fernández, C. (2011). Why do cities shrink? European Planning Studies, 19(8), 1375-1397. DOI: https://doi.org/10.1080/09654313.2011.593333 
Scanu, G., Madau, C., y Ugolini, G. M. (2006). Per un turismo nelle aree interne della Sardegna. Un primo approccio metodologico. En Sviluppo turistico e trasformazioni territoriali. Aree urbane, ecosistemi e complessità regionale (pp. 105-136). GRANTUR, Gruppo di Ricerca Nazionale sul Turismo.

Scanu, G., y Podda, C. (2016). Cartografia e turismo in aree rurali. Bollettino della Associazione Italiana di Cartografia, 158, 167-182. DOI: http://doi.org/10.13137/2282-572X/20746

Scanu, G., y Podda, C. (2018). Cartografia e sviluppo sostenibile. En M. Prezioso (Ed.), Capitale umano e valore aggiunto territoriale (pp. 93-117). Roma, Italia: Aracne.

Scanu, G. et al. (2018). Inner areas/periferie metropolitane e differenze con le Internal areas. En M. Prezioso (Ed.), Quale territorial impact assessment della coesione territoriale nelle regioni italiane. La concettualizzazione del problema (pp. 197-205). Bologna, Italia: Pàtron.

Scrofani, L., Novembre, C., y Petino, G. (2019). Le attività culturali e creative per il rilancio turistico delle aree interne in Sicilia. Il caso studio dell'Ypsigrock Festival. Bollettino della Associazione Italiana di Cartografia, 166, 28-42. DOI: http://doi.org/10.13137/2282-572X/30187

Storti, D. (2000). Tipologie di aree rurali in Italia. Roma, Italia: Istituto Nazionale di Economia Agraria (INEA). Recuperado de: http://antares.crea.gov.it:8080/documents/10179/152110/2316.pdf

Syssner, J. (2015). Planning for shrinkage? Policy implications of demographic decline in Swedish municipalities. Ager. Revista de Estudios sobre Despoblación y Desarrollo Rural, 7-31. DOI: http://doi.org/10.4422/ager.2015.14

Ugolini, G. M. (2004). Il rilancio delle aree rurali marginali: anche una questione di progetto culturale. En C. Madau (Ed.), Risorse culturali e sviluppo locale (pp. 67-62). Roma, Italia: Società Geografica Italiana.

Unión Europea (2016). Declaración de Cork 2.0 - Una vida mejor en el medio rural. Luxemburgo: Oficina de Publicaciones de la Unión Europea.

Recuperado de: https://enrd.ec.europa.eu/sites/enrd/files/cork-declaration es.pdf

UNWTO (2004). Rural tourism in Europe: Experiences, development and perspectives. Madrid: UNWTO. Recuperado de: https://www.e-unwto.org/doi/book/10.18111/9789284407163

Visvizi, A., y Lytras, M. D. (2018). It's not a fad: Smart cities and smart villages. Research in European and global contexts. Sustainability, 10(8), 2-10. DOI: https://doi.org/10.3390/su10082727

Visvizi, A., Lytras, M. D., y Mudri, G. (Eds.). (2019). Smart villages in the EU and beyond. Bingley, England: Emerald.

Xue, L., Kerstetter, D., y Hunt, C. (2017). Tourism development and changing rural identity in China. Annals of Tourism Research, 66, 170-182. DOI: http://doi.org/10.1016/j.annals.2017.07.016

\section{Sitiografía}

Las principales fuentes de datos proceden de los siguientes sitios web:

http://asc.istat.it/asc BL/

http://osservatorio.sardegnaturismo.it

http://www.regione.sardegna.it/speciali/programmasvilupporurale/leader/i-gal

https://ec.europa.eu/eurostat

https://opencoesione.gov.it/it/

https://www.agenziacoesione.gov.it

https://www.istat.it

https://www.politicheagricole.it/flex/cm/pages/ServeBLOB.php/L/IT/IDPagina/202

https://www.sardegnaautonomie.it

https://www.sardegnaprogrammazione.it 